\title{
Identification of Heat Shock Protein70 in Setaria digitata and its Evaluation as Diagnostic Marker for Lymphatic Filariasis
}

\section{Jayakumar K ${ }^{1 *}$, Sony Peter ${ }^{2}$ and Sarath Babu $\mathrm{K}^{3}$}

${ }^{1}$ Professor and Head, Department of Biochemistry, PMS College of Dental Science and Research, Vettapara, Thiruvanthapuram, Kerala, India

${ }^{2}$ Senior Lecturer, Department of Biochemistry, PMS College of Dental Science and Research, Vettapara, Thiruvanthapuram, Kerala, India

${ }^{3}$ Associate Professor, Department of Pharmacology, Dhanalakshmi Srinivasan

Medical College and Hospital, Perambalur, Trichy, Tamil Nadu, India

*Corresponding Author: Jayakumar K, Professor and Head, Department of Biochemistry, PMS College of Dental Science and Research, Vettapara,

Thiruvanthapuram, Kerala, India.
Received: July 21, 2020

Published: August 27, 2020

(C) All rights are reserved by Jayakumar K., et al.

\begin{abstract}
Heat shock protein 70 associated with lymphatic filariasis (LF) has been identified from a cattle filarial parasite Setaria digitata. A heat shock protein was detected in different life stages of Setaria digitata when exposed to an elevated temperature of $44^{\circ} \mathrm{C}$. A combination of ATP-agarose column chromatography and electro-elution was used for its purification from adult female extract. On closer examination, it migrated as a single band at $62 \mathrm{kDa}$ on $10 \%$ SDS-PAGE. These observations suggest a plausible biological connection of ScHSP70 with the disease and its strong immunogenic nature. ScHSP70 showed antigenic cross-reactivity with IgG class of antibody in different categories of filarial sera. However, when IgG subclasses were tested, IgG4 showed high specificity and sensitivity with symptomatic microfilaraemic sera.
\end{abstract}

Keywords: ATP-Agarose; Filarial Parasite; Heat Shock Proteins; Setaria digitata; W. bancrofti; SDS-PAGE

\section{Introduction}

In recent years numerous approaches have been employed for the identification of new diagnostic markers. Proteomics technologies offer unique chances to identify new candidate markers. Diagnostic markers for diseases like African trypanosomiasis, tuberculosis, cancer etc. have identified [1-3]. There is a constant need for new diagnostic markers with improved sensitivity and specificity for the screening of debilitating and dreadful diseases. Filariasis is a neglected tropical disease caused by parasitic worms called filariae. These are microscopic roundworms that dwell in the blood and tissues of humans, which are transmitted to humans by mosquitoes. The most important filarial diseases for humans are lymphatic filariasis, in which the adult worms are found in the lymphatic system. The lymphatic form of filariasis will be the focus of the site. Lymphatic filariasis is also referred to sometimes as "elephantiasis". Elephantiasis is actually an extreme clinical feature of filariasis. The painful and profoundly disfiguring visible the painful and profoundly disfiguring visible manifestations of the disease, lymphoedema, elephantiasis and scrotal swelling occur later in life and can lead to permanent disability. These patients are not only physically disabled, but suffer mental, social and financial losses contributing to stigma and poverty. In 2018, 893 million people in 
49 countries were living in areas that require preventive chemotherapy to stop the spread of infection. The global baseline estimate of people affected by lymphatic filariasis was 25 million men with hydrocele and over 15 million people with lymphoedema. At least 36 million people remain with these chronic disease manifestations [4] eliminating lymphatic filariasis can prevent. The painful and profoundly disfiguring visible Eliminating lymphatic filariasis can prevent unnecessary suffering and contribute to the reduction of poverty. Despite as researches on lymphatic filariasis continue, the focuses are on prevalence, treatment, prevention, transmission cycles, and even new species. However, current information on lymphatic filariasis is not complete, and further researches are still needed.

This fact actualizes that the effective initiatives like Mass Drug Administration (MDA) of DEC to an 'at risk population' needs a thorough screening at an early stage of the disease. The most intriguing and common sub-clinical condition of LF is the asymptomatic microfilaraemic individuals. It remains a major concern for the immune parasitologists due to lack of signs of the disease. In such incertitude and to successfully eliminate LF by the year 2020 , there is an urgent need to design and develop an accurate test for the early diagnosis of LF [5]. Hence, to identify novel candidates which can be exploited as possible markers in immunodiagnostic appears essential. Heat shock proteins (HSP) were discovered to be produced due to their increased expression in response to cellular aberrations such as elevated temperature, heavy metals and oxidative stress. Hence, to identify novel candidates which can be exploited as possible markers in immunodiagnosis appears essential. Heat shock proteins (HSP) were discovered to be produced due to their increased expression in response to cellular aberrations such as elevated temperature, heavy metals and oxidative stress. They are organized into their molecular size or function that includes HSP100, HSP90, HSP70, HSP60, HSP40 and small HSPs [6]. The most abundant and highly conserved HSPs are the HSP70 family members [8]. They are known to perform a range of vital cellular functions under stressed as well as in normal conditions to maintain the intracellular integrity and in adaptation and survival of the parasite within the hostile environment $[7,8]$. The fact that filarial parasite in its life cycle undergoes an inevitable thermal shift during migration between poikilothermic mosquito vectors and homeothermic vertebrate hosts. This shift results in producing a significant heat shock response to which the parasite counteracts to protect itself from aberrant conditions [8]. HSPs, as known to play an essential role in chaperon in functions are also being explored in recent times for their role in immune response.

\section{Materials and Methods}

Screening of HSPs in $S$. digitaita and somatic extract preparation.

Adult motile filarial parasite Setaria digitata were collected from the abdominal cavity of cattle (Bos indicus) from the local slaughter house of Thiruvananthapuram Corporation, Kerala. Adult female worms were used in this study because of larger in size than male and were incubated at $44^{\circ} \mathrm{C}$ for $3 \mathrm{~h}$ in KRB buffer. Somatic extract $(10 \%)$ was prepared by homogenizing adult worms in 50 $\mathrm{mM}$ phosphate buffer, $\mathrm{pH} 7.0$ and centrifuged at $12,000 \times \mathrm{g}$ for 30 minutes at $4{ }^{\circ} \mathrm{C}$. This supernatant was used as heat shocked adult female extract (hsAFE). The somatic extract of $S$. digitaita microfilariae (mf), exposed to $44^{\circ} \mathrm{C}$ was prepared according to the method described elsewhere [9]. Briefly, mf were subjected for sonication using ultrasonicator with a cycle of 30 s sonication at $20 \mathrm{KC}$ for 10 minutes followed by 30 s intervals in ice cold 50 mM PBS. The resultant was then centrifuged at $12,000 \times \mathrm{g}$ for 30 minutes at $4^{\circ} \mathrm{C}$. The clear supernatant was used as heat shocked mf extract (hsMFE). Adult female worms and its $\mathrm{mf}$ incubated at $37^{\circ} \mathrm{C}$, processed as described above were treated as control.

\section{Purification of ScHSP70}

ScHSP70 was purified using ATP-agarose column chromatography as described by Menoret [10] followed by electro-elution. Briefly, somatic extract of adult female $S$. digitaita was prepared in homogenization buffer (20 mM Tris-acetate, 1 mM PMSF, pH 7.5) and was loaded on to ATP-agarose column (Sigma, $1 \mathrm{~mL}$ gel bed) pre-equilibrated with buffer A $(20 \mathrm{mM}$ Tris-acetate, $20 \mathrm{mM} \mathrm{NaCl}$,

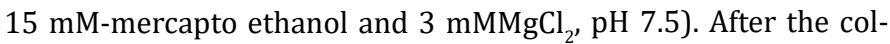
umn was washed with buffer $A$ and subsequently with $0.5 \mathrm{M} \mathrm{NaCl}$ in buffer A, HSPs were eluted with 3 mM ATP in buffer A. Protein migrated in SDS-PAGE having mol. wt. $62 \mathrm{kDa}$ was excised and further purified by electro-elution using $50 \mathrm{mM}$ phosphate buffer, $\mathrm{pH}$ 7.5.

\section{Protein estimation and SDS-PAGE}

Protein concentration was measured according to the dye binding method using bovine serum albumin as standard protein [11]. SDS-PAGE was performed under non-reducing conditions [12] and protein bands were visualized by Coomassie stain. Pre-stained mo- 
lecular weight markers (Sigma-Aldrich) were used for the estimation of subunit mass of purified ScHSP70.

\section{Antigenic cross-reactivity of ScHSP70 with $W$. bancrofti infect- ed human sera}

Antigenic cross-reactivity between purified ScHSP70 and various categories of $W$. bancrofti infected human sera was assessed using ELISA [13,14]. Briefly, 96-well micro plate (NUNC, USA) was coated with 100 micro liters of ScHSP70 (200 ng/well for IgG and IgG subclass assays). Different categories of filarial sera were used as primary antibody (1:100) and peroxidase conjugated anti human IgG (1:5000) or IgG1 and IgG4 (1:1000) as secondary antibody (Sigma-Aldrich). Color was developed using 0.05\% o-phenylenediamine (Sigma-Aldrich) as the substrate. Absorbance was measured at $490 \mathrm{~nm}$. Immuno blotting was performed as described by Lunde., et al [15]. Briefly, ScHSP70 was separated by SDS-PAGE, transferred onto nitrocellulose membrane (Sigma-Aldrich) and cut into strips. The strips were incubated with 1:100 diluted filarial sera (primary antibody) and subsequently with (secondary antibody) peroxidase conjugated anti-human IgG (1:5000) or IgG1 and IgG4 (1:1000) (Sigma-Aldrich). Protein bands were visualized using Di amino benzidine (Sigma-Aldrich) as the substrate.

\section{Selection of cases and collection of human sera}

Individuals were interviewed and examined for evidence of clinical filariasis (lymphoedema or elephantiasis). Microfilariae were detected by microscopic examination (MOTIC-B1 DMWB series, Germany) of stained thick blood films (100 micro liters) collected by finger prick method. Individuals were classified into three clinical groups as follows: persons with lymphoedema were considered to have clinical filariasis or chronic infection $(\mathrm{CH})$. None of the $\mathrm{CH}$ patients studied had microfilaraemia. Persons with microfilaraemia with no history or current evidence of lymphoedema were considered as microfilaraemics (MF). Endemic normal individuals (EN) were amicrofilaraemic and had no past history or current evidence of lymphoedema. Non-endemic normal, Ascariasis and Leishmaniasis (non-filarial) sera were kindly gifted by Filarial control unit of Nagercoil, Kanyakumari (Dist), Tamilnadu.

\section{Statistical analysis}

Mean, standard deviation and statistical significance were calculated using SPSS 10.0 version. Statistical significance was determined using Student's t-test and $\mathrm{p}$ value $\leq 0.005$ was considered significant. Experiments were performed in triplicate and the re- sults indicated are mean values unless otherwise mentioned.

\section{Results}

Screening of heat shock proteins in S. digitaita

SDS-PAGE of adult and microfilariae somatic extract revealed a significant difference between control and heat shocked worm extract. Interestingly, SDS-PAGE of both adult and mf extract afterheat shock showed four prominent protein bands of mol. wt. 123, 62, 51 and $32 \mathrm{kDa}$ which were either absent or very weak in case of control extract (Figure 1). Rests of the protein bands were very weak or almost invisible in heat shock extract.

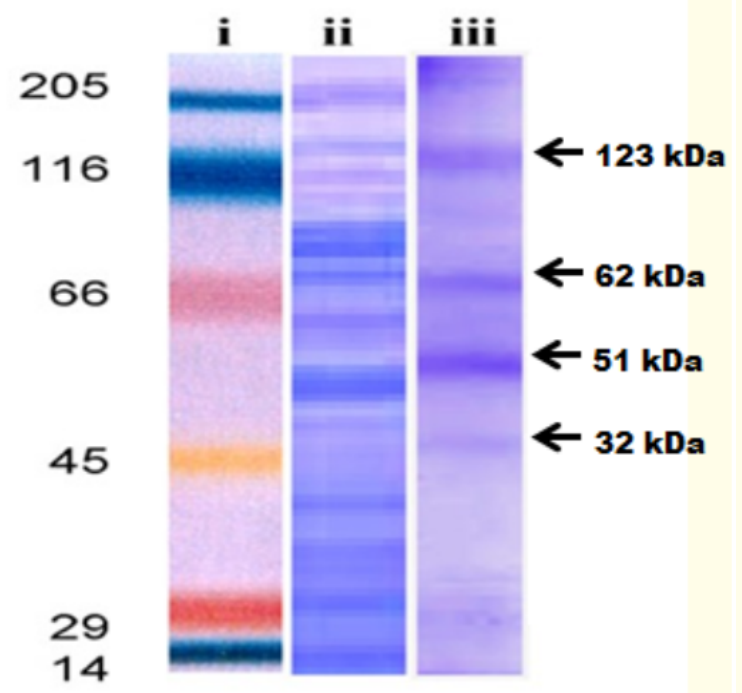

Figure 1: Screening and purification of heat shock proteins in S. digitata by $10 \%$ SDS-PAGE.

(i) Pre-stained mol. wt. marker, (ii) control adult female extract (AFE) and (iii) heatshocked adult female extract (hsAFE).

Recognition of $S$, digitaita antigens by IgG immunoblotting

Immunoblotting was performed to study the antigenic crossreactivityof $S$. digitaita antigens with W.bancrofti infected filarial human sera. Immunoblotting revealed a picture of potential S.digitata antigens, cross-reacting with various categories of filarialhuman sera. Interestingly, out of the 4 prominent proteinsof hsAFE, only 2 proteins having the molecular mass 123 and $62 \mathrm{kDa}$ showed crossreactivity with EN and MF sera respectively (Figure 2). 


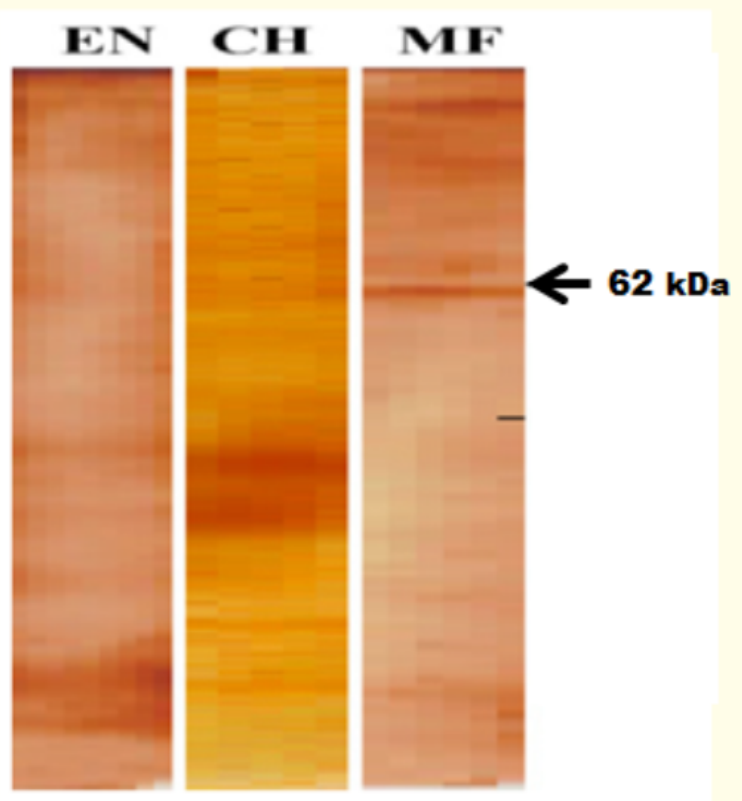

Figure 2: IgG immunoblotting of hsAFE with different categories of $W$. bancrofti infected human sera (The arrow indicates the position of $62 \mathrm{kDa}$ protein).

\section{Purification of ScHSP70}

ScHSP70 was purified to homogeneity using ATP-agarose column chromatography and electro-elution. After the column chromatography, SDS-PAGE revealed 3 protein bands. However, a single band of mol. wt. $62 \mathrm{kDa}$ was revealed after electro-elution (Figure 3).

\section{Antigenic cross-reactivity of purified ScHSP70 with}

\section{W.bancrofti infected human sera}

Antigenic efficacy of purified ScHSP70 against $W$. bancrofti infected filarial human sera was evaluated using ELISA and immunoblotting. ELISA showed a similar pattern of antigenic crossreactivity with different categories of filarial sera when studied for IgG (data not shown), IgG1 and IgG4 response. Reactivity with different categories of sera was in the order of $\mathrm{EN}<\mathrm{CH}<\mathrm{MF}$ while thereactivity with NEN, Ascariasis and Leishmaniasis/Kala-azar were found to be insignificant. However, cross-reactivity of ScHSP70 with MF sera remained exceedingly high when compared to other sera (both filarial and non-filarial) with IgG as well as IgG1 and i

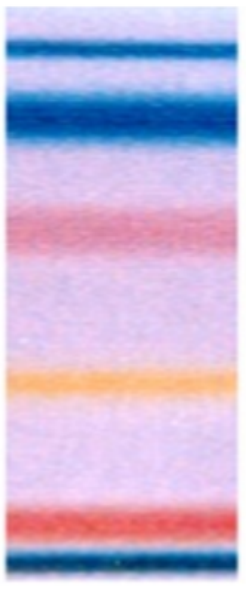

ii

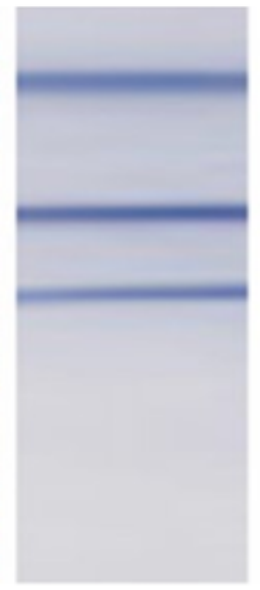

iii

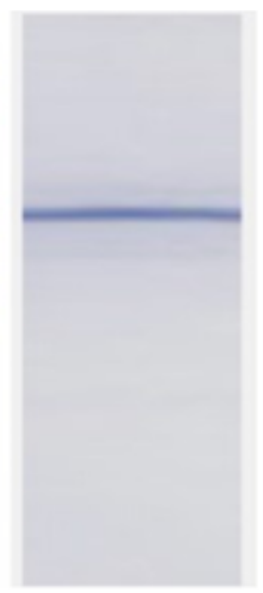

Figure 3: (i) Pre-stained mol. wt. marker, (ii) proteins eluted from ATP-agarose column chromatography and (iii) purified ScHSP70 after electro-elution.

IgG4. The statistical analysis showed that the antigenic crossreactivity of ScHSP70 with MF sera was more significant and specific with IgG4 subclass in comparison to other sera (Figure 4A and 4B). Results obtained were found to be statistically significant ( $\mathrm{p}$ value $\leq$ 0.005 ) when compared with non-endemic normal. The IgG4 immunoblotting of ScHSP70 with MF sera further explicates the specific antigenic cross-reactivity (Figure 5).

\section{Discussion}

HSPs, under physiological conditions are found as the intracellular proteins originally recognized due to their intense expression in response to elevated temperature, inflammation, irradiation, oxidizing agents, heavy metal ions and anoxia [16-22]. It was shown that the people who worked at high temperatures had a greater level of circulating HSP70 than who worked at normal temperatures [23]. This study has raised a possibility that HSP70 might be produced in response to heat or the development of heat shock [24]. Here, in our study too, the same result was imitated. Adult S.digitata and its $\mathrm{mf}$, when exposed to higher temperature, expressed higher levels of four prominent proteins of molecular mass 123, 62, 51 and $32 \mathrm{kDa}$, suggesting that these proteins might be critically required by the parasite for its survival under stress conditions. Out of the 

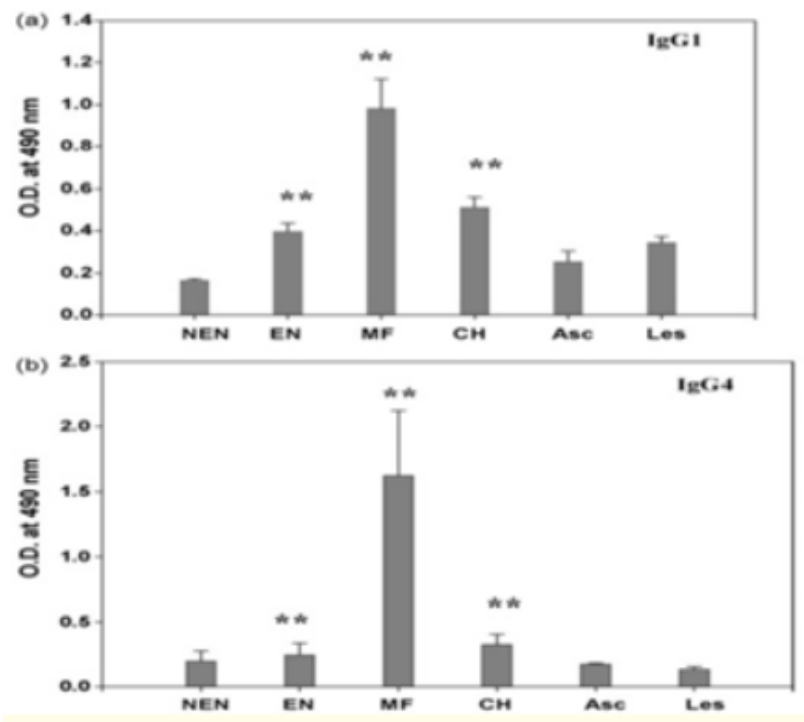

Figure 4: (a) and (b) Antigenic cross-reactivity of purified ScHSP70 with different categories of $W$. bancrofti infected human sera and non-filarial human sera via ELISA (a) IgG1 response and (b) IgG4 response. No. of sera used, $n=43$. EN: Endemic Normal; MF: Asymptomatic Microfilaraemic; $\mathrm{CH}$ : Chronic; NEN: Non-Endemic Normal; Asc: Ascariasis; Les: Leishmaniasis (Kala-azar). ${ }^{* *}$ p value $\leq 0.005$ was considered as significantly different.

four, $62 \mathrm{kDa}$ protein showed strong antigenic cross-reactivity exclusively with MF sera in immunoblotting. It appears that the expression of these proteins is develop mentally regulated as microfilarial stage also showed similar pattern after the heat shock. It has been already studied that the heat shock response enables the parasite to sustain in the hostile environment [25]. In Drosophila also, a close correlation was observed between induction of HSPs and its tolerance to high temperature where HSP70 was involvedin tolerance to extreme temperature [26]. ScHSP70 was purified to homogenecity using ATP-agarose affinity chromatography and electro-elution. The protein migrated as asingle band of $62 \mathrm{kDa}$ in SDS-PAGE. It was reported that the HSP70 family consists a number of highly conserved proteins which rangein size from 60 to 78 $\mathrm{kDa}[6,25]$. Binding of ScHSP70 to ATP-agarose column speculates that this protein might possess ATPase activity and belongs to HSP

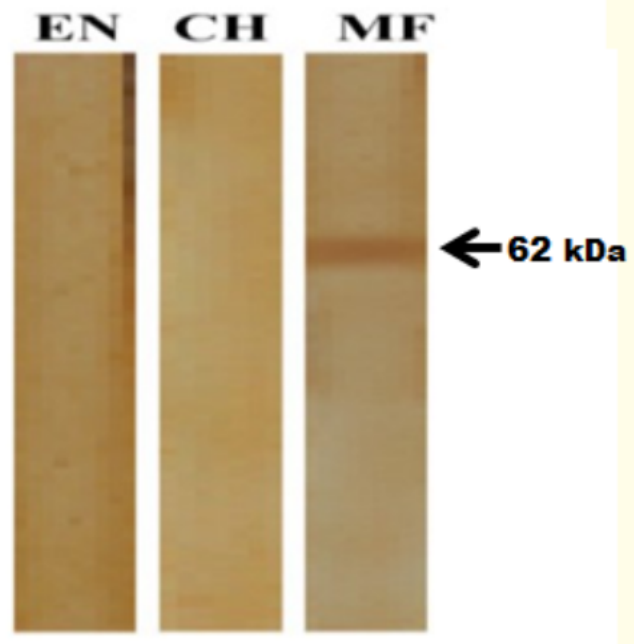

Figure 5: IgG4 immunoblotting of purified ScHSP70 with different categories of W.bancrofti infectedhuman sera.

family. Similar protocol was followed forthe purification of HSP70 from plants like Narcissus pseudo period on to pathogenic bacteria like Porphyromonas gingivalis, Actinobacillus actinomycetemcomitans and bacteroides forsythus and recombinant and endogenous HSP70 s $[27,28]$.

Antigenic cross-reactivity of ScHSP70 with $W$. bancrofti infected filarial human sera was evaluated using ELISA and immunoblotting assay. Many earlier studies using $S$. digitaita antigens have shown cross reactivity between the heterologous parasites [14,29,30-34]. These observations suggest that a protein homologous to $S$. digitaita HSP70 is also expressed by W. bancrofti and is thus exploited as a diagnostic marker in the screening of human lymphatic filariasis. It was reported that parasitic HSP70 and HSP90 represents a major target of immune response in parasitic infections were reported to be immune dominant antigens of the infecting organisms such as B. malayi, L. donovani, O. volvulus, S. mansoni, T. cruzi, P. falciparum etc [35]. The antigenic cross-reactivity with heterologous antigens is common in many tropical diseases, but the degree of specificity describes the suitability of such an antigen in detecting the disease by immunological methods [36]. Using techniques of diagnostic potential, such as ELISA and Western blotting circulating parasite antigens have been detected both in human and animal filariasis 
[38] by polyclonal anti sera. ScHSP70 pronounced a typical IgG response against $W$. bancrofti infected human sera. This depicts that ScHSP70 like antigen is exposed to the host immune system at every life stage of the parasite and sufficient levels of antibodies are also developed in the host. Filarial HSP70 is highly immunogenic as reported in case of B. malayi and Onchocerca volvulus [13,14]. The low specificity of ScHSP70 with IgG was improved significantly by employing IgG subclasses. It was reported in some earlier studies that the IgG4 levels have been associated with active infection in other helminthes infections such as filariasis and Onchocerciasis [39-41]. ScHSP70 showed high specificity towards IgG4 response with MF sera. A lucid demarcation of IgG4 response was observed not only when compared to the other sera (filarial and non-filarial) but also with IgG1 and IgG responses. This shows that the antibodies produced are more specific towards IgG4 which can be an indicator for the detection of asymptomatic microfilaraemic individuals. This is further substantiated when IgG4 response was studied using immune blotting that showed a stringent antigenic cross-reactivity with MF sera. As asymptomatic micro filaraemic stage is considered as one of the bottlenecks in the global elimination of LF, ScHSP70 can be promising agent for immune diagnosis. Also, due to unavailability of W. bancrofti and B. malayi, the bovine filarial parasite, $S$. digitaita can be a good source for heterologous antigens that can be explored as possible candidates for diagnosis, chemotherapy and vaccines.

\section{Conclusion}

We have shown that proteomic analysis is very useful in characterizing ScHSP70 as a disease-associated protein, although proteomics was performed in a small cohort. The results obtained in this study is promising, but further studies on recombinant expression are needed to determine its diagnostic accuracy in the immunodiagnostic of lymphatic filariasis in an endemic area. This study also opens a possibility to explore heat shock proteins for their use in the diagnosis of other helminthes.

\section{Acknowledgements}

The authors thankful to Filaria Control Unit of Kanyakumari District, Tamilnadu for their help.

\section{Conflict of Interest}

Authors do not have any conflict of interest for the present investigation.

\section{Bibliography}

1. Papadopoulos MC., et al. "A novel and accurate diagnostic test for human African trypanosomiasis". Lancet 363 (2004): 1358-1363.

2. Agranoff D., et al. "Identification of diagnostic markers for tuberculosis by proteomic fingerprinting of serum". Lancet 368 (2006): 1012-1021.

3. Susumu K., et al. "Identification by proteomic analysis of calreticulin as a marker for bladder cancer and evaluation of the diagnostic accuracy of its detection in urine". Clinical Chemistry 50.5 (2004): 857-866.

4. Kabatereine NB., et al. "How to (or not to) integrate vertical programmes for the control of major neglected tropical diseases in sub-Saharan Africa". PLoS Neglected Tropical Diseases 4.6 (2010): e755.

5. World Health Organization. "TDR Strategic Research: Lymphatic Filariasis" (2002).

6. Marissa VP., et al. "Inhibitors of heat shock response in biology and pharmacology". FEBS Letters 581 (2007): 3758-3769.

7. Jayasena SMT., et al. "Molecular characterization of a hsp70 gene from the filarial parasite Setaria digitata". International Journal of Parasitology 29 (1999): 581-591.

8. Gupta RS., et al. "Evolution of the HSP70 gene and its implications regarding relationship between archaebacteria, eubacteria and eukaryotes". Journal of Molecular Evaluation 37 (1993): 573-582.

9. Gupta S., et al. "MALDI mass sequencing and characterization of filarial glutathione-S-transferase". Biochemical Biophysical Research Communication 356 (2007): 381-385.

10. Menoret A. "Purification of recombinant and endogenous HSP70 s". Methods 32 (2004): 7-12.

11. Bradford MM. "A rapid and sensitive method for the quantization of microgram quantities of protein utilizing the principle of protein-dye binding". Annals Biochemistry 729 (1976): 248-254.

12. Newport G., et al. "Parasite heat shock proteins". Parasitology Today 4.11 (1988): 306-312. 
13. Laemmli UK. "Cleavage of structural proteins during the assembly of the head of bacteriophage T4". Nature 227 (1970): 680-685.

14. Rathaur S., et al. "Secretory acetylcholinesterases from Brugiamalayi adult and microfilarial parasites". Molecular and Biochemical Parasitology 26 (1978): 257-265.

15. Pokharel DR., et al. "Purification and characterization of a leucineaminopeptidase from the bovine filarial parasite Setaria cervi". Acta Tropica 106 (2008): 1-8.

16. LundeML., et al. "Filarial antigen in circulating immune complexes from patients with Wuchereria bancrofti filariasis". The American Journal of Tropical Medicine and Hygiene 38 (1988): 366-371.

17. Lindquist S. "The heat shock response". Annual Review of Biochemistry 55 (1986): 1151-1191.

18. Lydyard PM., et al. "Heat shock proteins: immunity and immunopathology". Immunology Today 11 (1990): 228-229.

19. Kaufmann SHE. "Heat shock proteins and the immune response". Immunology Today 11 (1990): 129-136.

20. Young RA. "Stress proteins and Immunology". Annual Review of Immunology 8 (1990): 401-420.

21. Young D., et al. "Molecular chaperones and the immune response". Philosophical Transactions Society B Biological Sciences 339 (1993): 363-368.

22. PollaBS., et al. "Differential induction of stress proteins and functional effects of heat shock in human phagocytes". Inflammation 19 (1995): 363-378.

23. Hyeyoung K. "Diagnostic significance of antibodies to heat shock proteins". Clinca Chemica Acta 337 (2003): 1-10.

24. HeXs., et al. "Detection of heat shock proteins by western blot cyberization". Clinical Journal of Hygiene 18 (1996): 396-399.

25. Miller MA., et al. "Inducible resistance to oxidant stress in the protozoan Leishmania chagasi. Inducible resistance to oxidant stress". Biological Chemistry 275 (2000): 33883-33889.

26. Li GC., et al. "Thermo tolerance in mammalian cells: a possible role for heat shock proteins". In: Atkinson BG, Walden DB, editors. Changes in eukaryotic gene expression in response to environmental stress. Orlando, FL: Academic Press (1985): 227-254.

27. Mayer MP., et al. "Hsp70 chaperones: cellular functions and molecular mechanism". Cell Molecular Life Science 6 (2005): 670-684.

28. Kaufmann SHE. "Heat shock proteins and the immune response". Immunology Today 11 (1990): 129-133.

29. Bonk M., et al. "Purification and characterization of chaperonin 60 and heat shock protein 70 from chromoplast of Narcissus pseudonarcissus". Plant Physiology 111 (1996): 931-939.

30. Hinode D., et al. "A general procedure for the isolation of heat shock proteins from periodonto pathogenic bacteria". Journal of Microbiology Methods 25 (1996): 349-355.

31. Kaushal NA., et al. "Identification of antigenic proteins of Setaria cervi by immunoblotting techniques". Immunolgical Investigations 16 (1987): 139-149.

32. Sharma S., et al. "Secretory acetyl cholinesterase of Setaria cervi microfilariae and its antigenic cross-reactivity with Wuchereria bancrofti". Tropical Medicine and International Health 3 (1998): 46-51.

33. Srivastava., et al. "Setaria cervi collagenase: IgG cleavage and inhibition by Wucheraria bancrofti infected sera". Acta Parasitology 49.3 (2004): 253-259.

34. Gupta S., et al. "Glutathione-S-transferase: immunoprophylactic potential against filarial parasite Brugiamalayi". Experimental Parasitology 109 (2005): 252-255.

35. Singh A. "Identification and characterization of a selenium dependent glutathione peroxidase in Setaria cervi". Biochemical Biophysical Research Communication 331 (2005): 1069-1074.

36. Kiang JG., et al. "Heat shock protein $70 \mathrm{kDa}$ : molecular biology, biochemistry and physiology". Pharmacololgy and Therapeutics 80.2 (1998): 183-120.

37. Kaliraj P., et al. "Detection of circulating filarial antigens in bancroftian filariasis". Indian Journal of Experimental Biology 17 (1979): 1148-1149. 
38. Hamilton RG., et al. "Immunoradiometric assay for quantitation of Dirofilariaimmitis antigen in dogs with heartworm infections". American Journal of Tertiary Research 45 (1984): 2055-2061.

39. Sugunan VS., et al. "Excretory-secretory antigens from a bovine filarial parasite cross react with human anti-filarial antibodies". Indian Journal Experimental Biology 28 (1990): 1124-1127.

40. Kwan-Lim GE., et al. "Filarial-specific IgG4 responses correlate with active Wuchereria infection". Journal Immunology 145 (1990): 4298-4305.

41. Lucius R., et al. "Specific and sensitive IgG4 immunodiagnosis of onchocerciasis with recombinant $33 \mathrm{kDa}$ Onchocerca volvulus protein (Ov33)". Tropical Medicine Parasitology 43 (1992): 139-143.

\section{Assets from publication with us}

- Prompt Acknowledgement after receiving the article

- Thorough Double blinded peer review

- Rapid Publication

- Issue of Publication Certificate

- High visibility of your Published work

Website: www.actascientific.com/

Submit Article: www.actascientific.com/submission.php

Email us: editor@actascientific.com

Contact us: +919182824667

Citation: Jayakumar K., et al. "Identification of Heat Shock Protein70 in Setaria digitata and its Evaluation as Diagnostic Marker for Lymphatic Filariasis". Acta Scientific Pharmaceutical Sciences 4.9 (2020): 15-22. 